
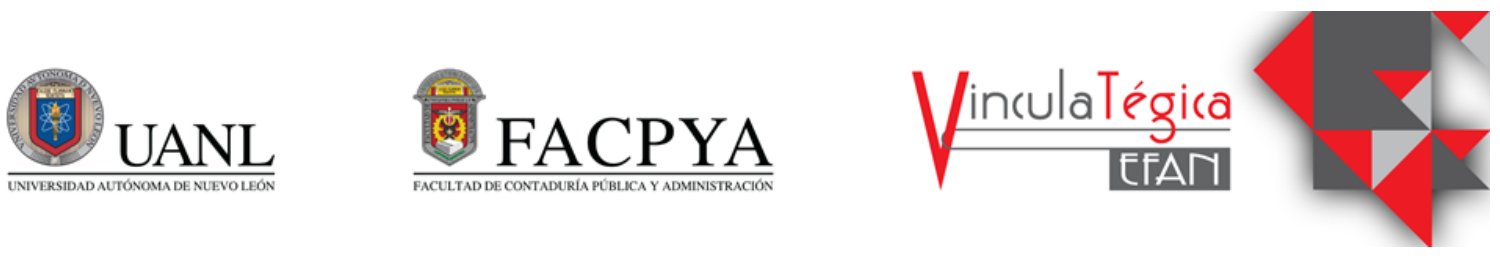

\title{
Medidas de creación de liquidez bancaria aplicadas en la banca múltiple mexicana
}

\author{
Juan Arturo Ruiz Valenciano ${ }^{1}$ y Klender Aimer Cortez Alejandro ${ }^{2}$ \\ ${ }^{1}$ Universidad Autónoma de Nuevo León, Facultad de Contaduría Pública y Administración \\ San Nicolás de los Garza, Nuevo León, México, juan.ruizv@uanl.edu.mx, Av. Universidad S/N Ciudad \\ Universitaria, (+52) 8183294000. \\ ${ }^{2}$ Universidad Autónoma de Nuevo León, Facultad de Contaduría Pública y Administración \\ San Nicolás de los Garza, Nuevo León, México, klender.cortezal@uanl.edu.mx, Av.Universidad S/N Ciudad \\ Universitaria, (+52) 8183294000 .
}

Información del artículo revisado por pares
Fecha de aceptación: junio-2021
Fecha de publicación en línea: diciembre-2021
DOI: https://doi.org/10.29105/vtga7.2-25

\section{Resumen}

El presente trabajo de investigación buscó describir las medidas de creación liquidez en la banca propuestas por Deep y Schaefer (2004) y Berger y Bouwman (2009), las cuales se fundamentan en la teoría de intermediación financiera, donde se hace mención que la creación de liquidez otorgada por los bancos es de suma importancia para las economías en las que interactúan. Ambas medidas fueron aplicadas a la banca múltiple mexicana, de manera consolidada con datos mensuales en un periodo comprendido de diciembre 2000 a diciembre 2020. Los resultados reportan una creación de liquidez a la baja, es decir, hay evidencia de una destrucción de liquidez por parte del gremio bancario en México.

Palabras clave: capital bancario, creación de liquidez, sistema bancario.

\section{INTRODUCCIÓN}

La teoría de intermediación financiera nos menciona que los bancos existen por dos principales razones, las cuales son la creación de liquidez y la transformación del riesgo (Bryany, 1980; Diamond y Dybvig, 1983; Deep y Schaefer, 2004; Berger y Bouwman, 2009, 2017; Pana, 2010; Le, 2019; Le y Pham, 2021, Dang, 2021).

\begin{abstract}
The present study describes the financial liquidity measures proposed by Deep \& Schaefer (2004) and Berger \& Bouwman (2009), which are focused on the liquidity provided by the banks and that is well-founded by financial brokering theory, where was mentioned that the liquidity created by the banks is crucial for interacting economies. Both measures were applied to the Mexican multiple banking, in a consolidated manner with monthly data between December 2000 to December 2020. The results report a downward creation of liquidity, that is, there is evidence of a destruction of liquidity by the Mexican banking system.
\end{abstract}

Keywords: banking system, capital bank, liquidity creation.

JEL: E51, E58.

En la actividad bancaria de creación de liquidez, según la teoría de intermediación financiera los bancos proveen de recursos a la economía, al financiar activos ilíquidos, como lo son préstamos comerciales y de consumo al público no bancario, esto lo realizan a través de la captación de pasivos líquidos, entre los cuales se encuentran depósitos a la vista o transacciones de depósitos institucionales 
(Bryan, 1980; Diamond y Dybing, 1983). La creación de la liquidez por parte de los bancos es de suma importancia para la macroeconomía ya que la estimulan para un mayor crecimiento económico (Detriagache y Rajan, 2008; Berger y Bouwman, 2017). En relación con la transformación del riesgo, los bancos transforman el riesgo a través de depósitos sin riesgo relativo, para el financiamiento de préstamos riesgosos (Diamond, 1984; Berger y Bouwman, 2009).

El presente trabajo busca explicar las medidas de liquidez propuestas por Deep y Schaefer (2004) y Berger y Bouwman (2009), aplicándolas en los resultados consolidados de las instituciones de banca múltiple del sistema financiero mexicano. Si bien, el modelo propuesto por Berger y Bouwman (2009) ha sido fundamental para trabajos más recientes como lo son Le y Pham (2021), Berger et al. (2019), entre otros que se mencionarán más adelante, el trabajo de Deep y Scheafer (2004) otorgaron una base al trabajo ya mencionado de Berger y Bouwman (2009) y sus cuatro medidas de liquidez, en donde nos centraremos para el desarrollo del trabajo de investigación en la medida de liquidez llamada "Cat fat".

El estudio de investigación mostrado en el presente artículo es de carácter descriptivo, ya que se busca interpretar y describir las medidas de liquidez propuestas por Deep y Schaefer (2004) y Berger y Bouwman (2009), junto a su clasificación de los conceptos del balance general y fuera del mismo de las instituciones de banca múltiple dentro del sistema financiero mexicano. El análisis se realiza en el periodo comprendido de diciembre 2000 a diciembre 2020, con una muestra consolidada de la banca múltiple de 241 meses con datos obtenidos de la Comisión Nacional Bancaria y de Valores (CNBV).

Con la revisión de la literatura se plantea la hipótesis H1, la cual se representa de la siguiente manera:

H1: La Banca múltiple mexicana crea liquidez como medida de producción del sector bancario.

La hipótesis contempla el enfoque de la creación de liquidez como producción al otorgamiento de recursos a través de préstamos a los agentes en la economía mexicana y la transformación de los activos ilíquidos a pasivos líquidos, con el fin de un desarrollo económico.

Los resultados obtenidos con las medidas de liquidez propuestas por Deep y Schaefer (2004) y Berger y Bouwman (2009) mostraron una creación muy baja de liquidez y una destrucción de liquidez respectivamente, dentro del periodo considerado en el estudio.

El resto del presente artículo se compone del marco teórico de la creación de liquidez bancaria y su revisión de la literatura previa a ambos modelos de medición, siguiendo con la descripción teórica de los modelos de liquidez. Posterior se detalla el método de la segmentación de los conceptos del balance para las medidas de liquidez y su aplicación en la banca múltiple mexicana. Llegando a los resultados obtenidos de la creación de liquidez por parte de la consolidación de la banca múltiple mexicana en el presente trabajo, para así emitir nuestras conclusiones respecto a ambas medidas de liquidez obtenidas de la revisión de la literatura, interpretando una fragilidad en la creación de liquidez por parte de las instituciones como transformadoras de liquidez, como lo menciona Deep y Schaefer (2004).

\section{MARCO TEÓRICO}

En la presente sección se busca contextualizar la creación de liquidez junto a la intermediación financiera y como los bancos crean liquidez al financiar préstamos a los agentes económicos. Además, se revisarán las dos medidas de creación de liquidez propuestas por Deep y Schaefer (2004) y Berger y Bouwman (2009) respectivamente, junto a su enfoque de medida de la liquidez proporcionada por los bancos. Posteriormente, haremos mención los principales modelos propuestos, por diferentes autores, que han utilizado las medidas de creación de liquidez revisadas en el presente trabajo de investigación.

\subsection{La intermediación financiera y la creación de liquidez}

$\mathrm{La}$ intermediación financiera nos menciona 
con la interpretación de Diamon y Dybvig (1983), que las entidades financieras (bancos principalmente), se financian al captar recursos a través de instrumentos de depósitos a la vista. Los cuales son establecidos en el lado de los pasivos del balance y son denominados pasivos líquidos.

Esto da la oportunidad que los recursos captados sean colocados a través de ciertos préstamos comerciales a empresas o gobiernos, o bien, préstamos de consumo como los son, tarjetas de crédito, créditos personales y créditos a la vivienda, por mencionar algunos, los cuales son registrados del lado de los activos del balance y son denominados activos ilíquidos. Creando a grandes rasgos liquidez en la economía en la que interactúan. (Diamon y Dybvig 1983; Deep y Schaefer 2004; Allen y Carletty 2009; Berger y Bouwman 2009; Le 2019).

Cómo se mencionó en los párrafos anteriores, la intermediación financiera se encarga principalmente en la creación de liquidez, ya que es una razón clave de la existencia de los bancos en la economía (Berger y Bouwman, 2017). De manera más puntual, los bancos crean está liquidez al otorgar créditos (activos no líquidos) a empresas o al público en general a partir de pasivos líquidos (Berger y Bouwman, 2017). $\mathrm{O}$ bien, en transformar activos ilíquidos en pasivos líquidos (Berger et al. (2019). Es decir, la liquidez se crea al financiar 1 dólar de activos no líquidos con 1 dólar de pasivos líquidos, es ahí donde se crea la máxima liquidez. Sin embargo, en contra parte la liquidez se destruye al otorgar 1 dólar de pasivos líquidos en financiar 1 dólar de activos líquidos como valores bursátiles o 1 dólar de capital para financiar 1 dólar de activos líquidos (Berger y Bouwman, 2017).

\subsubsection{El capital bancario}

Una de las preguntas que se han realizado los economistas y organismos reguladores, ha sido ¿qué papel tiene el capital bancario para la operación de las instituciones bancarias? (Diamond y Rajan, 2000). Existe una extensa literatura acerca del capital bancario y como irrumpe la creación de liquidez en la economía.
Las primeras teorías de intermediación financiera sugieren que los bancos no cuentan con capital, esto debido a como se comentó en los párrafos anteriores, una de las principales actividades es la captación de recursos a través de depósitos y colocándolos a través de préstamos a los agentes económicos (Berger y Bouwman, 2017). Sin embargo, en trabajos más recientes, el capital bancario ha sido incluido por diferentes motivos, ya que se estima que, al momento de contar con capital, los bancos pueden llegar a absorber un mayor nivel de riesgo (Berger y Bouwman, 2017), el cual puede hacerlos un poco más fuertes en caso de un escenario de falta de liquidez interna del banco al estar asociado al riesgo de liquidez y el riesgo de impagos.

La inclusión de ambos enfoques de incluir o no el capital en la teoría, ha generado ideologías contradictorias sobre la creación de liquidez por parte de los bancos, ya que como se mencionó, el contar con un grado de capital propio por parte de los bancos puede ayudar a disminuir el riesgo (Coval y Thakor, 2005), sin embargo, esto no ha sido comprobado del todo, ya que los trabajos de Allen y Gale (2004) hacen mención que al incurrir en realizar prestamos más riesgosos, se ha visto afectada la creación de liquidez, por las pérdidas asociadas a estos préstamos.

\subsection{Medida de creación de liquidez de Deep y Schaefer (2004)}

Deep y Schaefer (2004) nos mencionan en concordancia con la teoría de la intermediación financiera, que la principal función de la creación de la liquidez es aceptar depósitos y canalizarlos a través de préstamos ilíquidos a largo plazo, afectando directamente el ahorro para canalizarlo hacia la inversión, jugando un papel muy útil y necesario para el desarrollo económico.

Para esto, hacen mención que es primordial lograr encontrar una medida de creación de liquidez, proponiendo en su modelo la brecha de transformación de liquidez (LT). La cual la describen como la diferencia entre los pasivos líquidos y los activos mantenidos entre los activos totales de un banco. Planteando que un banco financiado 
en su mayoría por depósitos líquidos, que realiza prestamos ilíquidos en su mayoría y cuenta con una minoría de activos líquidos, presenta una mayor creación de liquidez (Deep y Schaefer, 2004).

El cálculo de la brecha de creación de liquidez (LT) se describe en la Ecuación (1), donde, los activos líquidos son denominados (L), activos ilíquidos como préstamos son llamados (I), dando el total de activos como L $+\mathrm{I}=\mathrm{A}$, esto por el lado de los activos. En cuanto a los pasivos y el capital, los depósitos pasivos líquidos, denominados como (D) y pasivos a largo plazo como (B), el capital como (E), dando de manera que $\mathrm{D}+\mathrm{B}+\mathrm{E}=\mathrm{A}$ (Deep y Schaefer, 2004).

$$
\text { GAP LT }=\frac{D-L}{A}
$$

donde:

GAP LT $=$ Brecha de creación de Liquidez

$\mathrm{D}=$ Pasivos líquidos

$\mathrm{L}=$ Los activos líquidos de un banco

$\mathrm{A}=$ Activos totales

De tal manera que la brecha se encuentra entre -1 y 1 , donde 1 es la máxima creación de liquidez y -1 la destruye, concordando con la teoría de liquidez.

Los autores segmentaron los registros de activos y pasivos de la siguiente manera. Activos líquidos como efectivo, saldos de instituciones depositarias, valores gubernamentales y privados. Pasivos líquidos, depósitos de disponibilidad inmediata, y depósitos empresariales, fondos federales, pasivos comerciales y depósitos a plazo con vencimiento menor a 1 año, (Deep y Schaefer, 2004).

El trabajo de Deep y Schaefer (2004), se enfocó en la banca comercial estadounidense, a través de 200 bancos en el periodo comprendido del segundo trimestre de 1997 al segundo trimestre de 2001, con los reportes trimestrales solicitados por el organismo regulador.

El periodo de estudió arrojo como resultado que los bancos estadounidenses tenían una brecha de creación de liquidez bastante baja con una media de 0.20, recordando que es un indicador que está entre -1 y 1 , donde la máxima liquidez es 1 . Una desviación estándar de 0.16 según Deep y Schaefer (2004), concluyendo como ya se mencionó la creación de liquidez por parte de los bancos estadounidenses es poca o nula con respecto a la medición de brecha de liquidez.

\subsection{Medida de creación de liquidez de Berger y Bouwman (2009)}

La ideología de la teoría moderna de intermediación financiera menciona que los bancos cumplen dos funciones principales, la creación de liquidez y transformar el riesgo. Berger y Bouwman (2009) identificaron que la mayoría de los estudios se encontraban basados en la transformación del riesgo y que no existían medidas de creación de liquidez que ayudara al cálculo del desarrollo de la banca en la economía.

$\mathrm{Su}$ trabajo se centró en realizar medidas integrales de creación de liquidez en la economía, esto con el fin de ver el desarrollo de la banca y en la creación de liquidez y como el capital bancario afecta o no a la liquidez.

El modelo constó de tres pasos donde se clasificaron los conceptos del balance general y fuera del balance como primer paso, en el segundo paso se asignaron ponderaciones a los conceptos y en el tercer paso construyeron 4 medidas de creación de liquidez, con las diferentes combinaciones realizadas en el paso uno, denominándolas "Cat fat", "Cat nonfat", "mat fat" y "Mat non fat" (Berger y Bouwman, 2009).

En el primer paso, se clasificaron los conceptos dentro y fuera del balance en función de con que tanta facilidad en tiempo y costo pueden convertirse los compromisos y los recursos en efectivo para así cubrir las necesidades de liquidez en la institución bancaria, clasificados como líquidos, semilíquidos e ilíquidos de los cuales se describen a continuación en la tabla 1 (Berger y Bowman, 2009).

Tabla 1. Clasificación de conceptos del balance, Berger y Bouwman (2009). 


\begin{tabular}{|c|c|c|}
\hline Activos* & Pasivos* & $\begin{array}{c}\text { Capital* } \\
\text { Fuera del balance } \\
\end{array}$ \\
\hline No líquidos (1/2) & \multirow{2}{*}{$\begin{array}{c}\text { No Líquidos (-1/2) } \\
\text { Responsabilidad del banco en las aceptaciones } \\
\text { bancarias }\end{array}$} & Garantías no líquidas (1/2) \\
\hline Préstamos Inmobiliarios comerciales & & Cartas de Crédito en stand by \\
\hline Préstamos Agricultura & Deuda subordinada & Compromisos no utilizados \\
\hline Préstamos comerciales e industriales & \multirow[t]{2}{*}{ Otros Pasivos } & Cartas de crédito comerciales y similares \\
\hline $\begin{array}{c}\text { Otros préstamos y arrendamiento mayores a } \\
1 \text { año } \\
\text { Activos intangibles } \\
\text { Otros activos }\end{array}$ & & Todos los demás pasivos fuera de balance \\
\hline Semilíquidos (0) & Semilíquidos (0) & Garantías semilíquidas (0) \\
\hline Préstamos inmobiliarios residenciales & Depósitos a Plazo & Derivados de crédito neto \\
\hline Créditos de consumo & Otro dinero prestado & Valores netos prestados \\
\hline $\begin{array}{l}\text { Préstamos a institución depositaria } \\
\text { Préstamos al gobierno estatales y locales } \\
\text { Préstamos a gobiernos extranjeros }\end{array}$ & $\begin{array}{l}\text { Fondos federales comprados de un día para otro } \\
\text { Pasivos comerciales }\end{array}$ & \\
\hline Arrendamiento $<=1$ año & & \\
\hline Líquidos $(-1 / 2)$ & Líquidos (1/2) & Garantías liquidas/Derivados líquidos (-1/2) \\
\hline Efectivo y adeudos de otra institución & Depósitos de transacciones & Participaciones netas adquiridas \\
\hline Todos los valores accionarios & Depósitos de ahorro & Derivados de tipos de interés \\
\hline Activos comerciales & Fondos federales comprados de un día para otro & Derivados de tipo de cambio \\
\hline Fondos federales vendidos & Pasivos comerciales & Derivados de acciones y materias primas \\
\hline
\end{tabular}

Fuente: elaboración propia recolectada de Berger y Bouwman (2009).

* Los pesos asignados se presentan en paréntesis.

En el segundo paso los autores asignaron ponderación a las clasificaciones realizadas en el punto anterior conforme a la teoría de liquidez. Estas ponderaciones constaban de $1 / 2,0$ y $-1 / 2$, las cuales se muestran en la pasada tabla 1 marcadas entre paréntesis. Determinando que las ponderaciones se basan en suma de dólar por dólar, ya que, la liquidez máxima se crea cuando los bancos transforman 1 dólar de activos ilíquidos en 1 dólar de pasivos líquidos. Y se destruye cuando 1 dólar de activos líquidos en 1 dólar de pasivos no líquidos (Berger y Bowman 2009).

Continuando con el párrafo anterior, citan un ejemplo donde se crea la máxima liquidez conforme a su modelo, planteando que 1 dólar financiado en activos ilíquidos de 1 dólar pasivos líquidos se crea la máxima liquidez equivalente a $1 / 2 * 1+1 / 2 * 1=1$ (Berger y Bowman 2009).

En el tercer paso de la creación de medidas de liquidez de Berger y Bouwman (2009) combinaron las actividades segmentadas en los pasos anteriores con el fin de determinar la madurez de los activos y su categoría, dentro y fuera del balance, determinando 4 medias de creación de liquidez, "Cat fat", "Cat nonfat", "mat fat" y "Mat non fat". Donde destacan la medida de liquidez "Cat Fat" como integral creación de liquidez independientemente del vencimiento.
El estudio de Berger y Bouwman (2009) contemplo de muestra prácticamente todos los bancos comerciales de estados unidos, con un cierto criterio de exclusión por diferentes motivos, como es, no tiene prestamos pendientes, o que no tengan capital social. Durante un periodo de 10 años de 1993 a 2003, en base a los informes anuales publicados por las instituciones.

Los resultados que obtuvieron Berger y Bowman (2009) respecto a las medidas de liquidez, dio que los bancos más grandes mostraron una correlación positiva en la creación de liquidez con respecto al capital del banco en medidas que incluían actividades fuera del balance, para bancos pequeños y medianos resulto una correlación negativa con respecto a las medidas de creación de liquidez versus el capital. Otro punto que arrojo el estudio es que la banca estadounidense arrojo una creación de liquidez de 4.6 dólares por cada dólar de capital.

\subsection{Literatura de modelo de creación de liquidez}

La creación de liquidez es un factor de crecimiento para las economías, sin embargo, la creación excesiva de liquidez bancaria puede ser parte de un efecto desfavorable ya que puede generar burbujas que estallen y aumenten la probabilidad de una crisis financiera. Probablemente dada esta 
ambigüedad, se ha desarrollado una extensa literatura sobre como la creación de liquidez ha afectado la economía de manera positiva o negativa.

A continuación, revisaremos algunos estudios que han realizado a través de las medidas de liquidez expuestas en el presente trabajo.

Como se mencionó en los apartados anteriores el trabajo de Deep y Schaefer (2004), propuso en cierta medida el desarrollo de las medidas de creación de liquidez por parte de Berger y Bouwman (20099, que se ha venido explicando en el cuerpo del documento. Esto sirvió para analizar diferentes enfoques de cómo la creación de liquidez se ve afectada por variaciones en la incertidumbre de la política económica en el trabajo de Pastor y Veronesi (2013) y Kelly y Veronesi (2016).

Fungáčová et al. (2017) enfocan su modelo en la medición de creación de liquidez en el sistema bancario ruso. Analizando como afecta el seguro de depósitos, el capital bancario y la liquidez. Encontraron que no hay evidencia significativa en la implementación del seguro en esta relación, cuando se consideran todos los bancos. Sin embargo, detectaron que los bancos con mayor riesgo de insolvencia por descapitalización disminuyen el beneficio del capital a la creación de liquidez aun con la implementación del seguro.

El trabajo de Berger et al. (2019) analiza la creación de liquidez de la banca islámica a través del modelo de Berger y Bouwman (2009), concluyendo que los bancos islámicos crean más liquidez por unidad de activos en general en el lado de los activos, y dando resultados de generación de liquidez menor en el lado del pasivo por unidad de activos generales y fuera del balance que los bancos convencionales.

Le y Pham (2021) investigaron sobre la relación entre la liquidez, el capital bancario y la probabilidad de impago en las economías emergentes de Asia, donde encontraron que el capital bancario y el riesgo están correlacionadas de manera positiva posterior a controlar la creación de liquidez y una relación negativa del riesgo de crédito con la creación de liquidez. Concluyendo que, los resultados son bidireccionales negativos con la creación de liquidez y capital.

Como se observa en la sección, la ideología presentada por la intermediación financiera es de suma importancia para el desarrollo económico, al proveer de liquidez a los agentes económicos a través de los préstamos. Sin embargo, el riesgo asociado al incumplimiento de los préstamos, riesgo de mercado o incertidumbre de la política económica (como tasas de interés), han llevado a cabo ideologías con un enfoque al requerimiento de capital base para las instituciones bancarias, contraponiendo puntos a favor y en contra de este recurso.

Es por esta razón que la elaboración de medidas de liquidez es una base requerida para evaluar la efectividad de la industria bancaria en las economías y como afecta los diferentes escenarios mencionados en los puntos anteriores, como una incertidumbre de la política económica.

\section{MÉTODO}

El presente trabajo de investigación por sus características se encuentra realizado bajo un método del tipo cuantitativo con un alcance descriptivo, ya que como se mencionó, se buscó interpretar los modelos propuestos de creación de liquidez bancaria por Deep y Schaefer (2004) y Berger y Bouwman (2009), y aplicarlos a la banca múltiple mexicana.

La información fue obtenida en los portales de la Comisión Nacional Bancaria y de Valores, catálogo de reportes. La cual es el organismo regulador del sistema financiero mexicano. La información se encuentra consolidada bajo los estándares de este organismo.

El periodo de estudio comprende de diciembre 2000 a diciembre de 2020 , con un total de observaciones de 241 meses. La muestra de los datos es la representación total consolidad de la banca múltiple mexicana con la segmentación de los conceptos del balance general conforme a los modelos de medición de creación de liquidez propuesto por Deep y Schaefer (2004) y Berger y Bouwman (2009), los 
cuales se describirán en la presente sección.

El procesamiento de la información será a través de estadística descriptiva, para tener una noción de los resultados obtenidos de ambas medidas de liquidez del consolidado de la banca múltiple mexicana.

A continuación, se mostrará la segmentación del balance publicado por la CNBV, de manera propuesta por ambos autores.

\subsection{Segmentación del balance según Deep y Schaefer (2004).}

Como se observó en el marco teórico, la principal función de la creación de liquidez, es la de captar recursos a través de depósitos y colocarlos a los agentes económicos por medio de préstamos activos ilíquidos. Es por esta razón que Deep y Schaefer (2004) segmentaron los conceptos del balance en activos líquidos, activos ilíquidos, pasivos ilíquidos. A continuación, en la tabla 2, utilizado como base el modelo de brecha de liquidez (GAP LT) de Deep y Schaefer (2004), se segmentaron los rubros del balance general en el reporte proporcionado de manera mensual por parte de la Comisión Nacional Bancaria y de Valores. Los cuales fueron aplicados para la creación de la brecha de liquidez en la banca múltiple mexicana.

Tabla 2. Clasificación de conceptos del balance Deep y Schaefer (2004) con información de la CNBV.

\begin{tabular}{ccc}
\hline Activos* & Pasivos* & Capital* \\
\hline Estimación preventiva para riesgos crediticios & Líquidos \\
Líquidos & Depósitos de exigibilidad inmediata \\
Inversionibilidades en valores & Cuenta global de captación sin movimientos \\
Operaciones con valores y derivados \\
Operaciones con valores y derivados \\
Otros activos & $\begin{array}{c}\text { Préstamos interbancarios y de otros organismos } \\
\text { Obligaciones subordinadas en circulación } \\
\text { Otros pasivos } \\
\text { No Líquidos }\end{array}$ & No líquidos \\
Créditos Empresas Vigente & Depósitos a plazo y títulos de crédito emitidos \\
Créditos Entidades financieras Vigente & \\
Créditos Entidades gubernamentales Vigente & \\
Créditos Consumo Vigente & \\
Créditos Vivienda Vigente & \\
Créditos Empresas Vencido & \\
Créditos Entidades financieras Vencido & \\
Créditos Entidades gubernamentales Vencido & \\
Créditos Consumo Vencido & \\
Créditos Vivienda Vencido & \\
\hline
\end{tabular}

Fuente: elaboración propia recolectada de Deep y Schaefer (2004).

\subsection{Segmentación del balance según Berger y Bouwman (2009)}

Para Berger y Bouwman (2009), era primordial realizar medidas integrales de creación de liquidez proponiendo 4 medidas. En la presente sección segmentaremos los rubros del balance general publicado por la CNBV conforme a lo propuesto por Berger y Bouwman (2009), posterior explicaremos la medida "CAT FAT", la cual es la que se utilizó en el presente estudio, por ser la medida preferida por los autores por motivo que contempla la creación de liquidez de manera total.

En la tabla 3 se muestra la segmentación en base al modelo propuesto por Berger y Bouwman (2009) del balance general el cual es publicado por la CNBV de manera mensual, mostrando la situación financiera de la banca múltiple mexicana.

Una vez clasificado los conceptos del balance, la medida propuesta como "CAT FAT" por Berger y Bouwman, es la medida de creación de liquidez que incluye los conceptos de préstamos, es decir, en comerciales, de consumo de vivienda, y no por fecha de vencimiento. A continuación, mostraremos el cálculo de la medida de liquidez CAT FAT:

$$
\begin{aligned}
\text { CatFat }= & (1 / 2 *(\mathrm{AI}+\mathrm{PL}))+ \\
& (0 *(\mathrm{AS}+\mathrm{PS}))+ \\
& \left(-1 / 2^{*}(\mathrm{AL}+\mathrm{PI}+\mathrm{Cap})\right)
\end{aligned}
$$


En donde:

$\mathrm{AI}=$ Activos ilíquidos

$\mathrm{PL}=$ Pasivos líquidos

$\mathrm{AS}=$ Activos semilíquidos

PS = Pasivos semilíquidos

$\mathrm{AL}=$ Activos líquidos

$\mathrm{PI}=$ Pasivos ilíquidos
Cap $=$ Capital

A través de las medidas mencionadas en la presente sección, se llevó a cabo la valoración de la banca múltiple mexicana, donde revisaremos los resultados en el siguiente apartado.

Tabla 3. Clasificación de conceptos del balance Berger y Bouwman (2009), con información de la CNBV.

\begin{tabular}{|c|c|c|}
\hline Activos* & Pasivos* & Capital* \\
\hline Líquidos $(-1 / 2)$ & Líquidos $(1 / 2)$ & Capital $(-1 / 2)$ \\
\hline Disponibilidades & Depósitos de exigibilidad inmediata & Capital contable \\
\hline Inversiones en valores & Cuenta global de captación sin movimientos & $\begin{array}{l}\text { Estimación preventiva para } \\
\text { riesgos crediticios }\end{array}$ \\
\hline \multicolumn{3}{|l|}{ Operaciones con valores y derivados } \\
\hline Semilíquidos $(0)$ & Semilíquidos (0) & \\
\hline Créditos Consumo Vigente & Depósitos a plazo y títulos de crédito emitidos & \\
\hline Créditos Vivienda Vigente & Operaciones con valores y derivados & \\
\hline Créditos Consumo Vencido & Obligaciones subordinadas en circulación & \\
\hline \multicolumn{3}{|l|}{ Créditos Entidades gubernamentales Vigente } \\
\hline \multicolumn{3}{|l|}{ Créditos Vivienda Vencido } \\
\hline \multicolumn{3}{|l|}{ Créditos Entidades gubernamentales Vencido } \\
\hline No Líquidos $(1 / 2)$ & No líquidos $(-1 / 2)$ & \\
\hline Créditos Empresas Vigente & Préstamos interbancarios y de otros organismos & \\
\hline Créditos Entidades financieras Vigente & Otros pasivos & \\
\hline Créditos Empresas Vencido & & \\
\hline Créditos Entidades financieras Vencido & & \\
\hline
\end{tabular}

Fuente: Elaboración propia con información de la Comisión Nacional Bancaria y de Valores (CNBV)

* Los pesos asignados se presentan en paréntesis.

\section{RESULTADOS}

La teoría de intermediación financiera menciona que los bancos existen para cumplir con dos funciones primordiales, donde la primera es proveer de liquidez a los agentes económicos y la segunda transformar el riesgo (Diamond y Dybvig 1983). La primera es de suma importancia ya que al proveer de liquidez a la economía esto impulsa un mayor crecimiento. Por otra parte, la transformación del riesgo la realizan al captar depósitos sin riesgo, para colocar créditos con un nivel de riesgo (Diamon 1983). Para esto Deep y Schaefer (2004) y Berger y Bouwman (2009), ha propuesto sus medidas de liquidez, las cuales fueron aplicadas a la banca múltiple mexicana, en donde arrojaron los siguientes resultados.

En cuanto a la medida propuesta por Deep y Schaefer (2004) la banca múltiple mexicana mostro un GAP LT promedio de 0.1076 y una desviación estándar de 0.0628 , mostrada en la Tabla 4.

Tabla 4. Resultados de GAP LT Deep y (2004) con información de la CNBV, periodo comprendido de diciembre 2000 a diciembre 2021.

\begin{tabular}{cc}
\hline Estadístico & GAP LT \\
\hline Media & 0.1076 \\
Error típico & 0.0040 \\
Mediana & 0.0865 \\
Moda & $\#$ N/A \\
Desviación estándar & 0.0628 \\
Varianza de la muestra & 0.0039 \\
Curtosis & -1.4202 \\
Coeficiente de asimetría & 0.2388 \\
Rango & 0.2000 \\
Mínimo & 0.0120 \\
Máximo & 0.2119 \\
Suma & 25.9310 \\
Cuenta & 241 \\
\hline
\end{tabular}

Fuente: Elaboración propia, con información de la Comisión Nacional Bancaria y de Valores (CNBV).

Para la medida 
Cat Fat propuesta por Berger y Bouwman (2009), los resultados que arrojaron el consolidado de la banca múltiple mexicana fue una creación (destrucción) de liquidez promedio de $-843,803$ millones de pesos, con una desviación estándar de 242,517, los cuales se muestran en la Tabla 5.

Tabla 5. Resultados de Cat Fat Berger y Bouwman (2009) con información de la CNBV, periodo comprendido de diciembre 2000 a diciembre 2021.

\begin{tabular}{cc}
\hline Estadístico & Cat Fat \\
\hline Media & $-843,803$ \\
Error típico & 15,622 \\
Mediana & $-876,620$ \\
Moda & $\# \mathrm{~N} / \mathrm{A}$ \\
Desviación estándar & 242,517 \\
Varianza de la muestra & $58,814,679,702$ \\
Curtosis & -1 \\
Coeficiente de asimetría & 0 \\
Rango & 994,713 \\
Mínimo & $-1,288,597$ \\
Máximo & $-293,884$ \\
Suma & $-203,356,534$ \\
Cuenta & 241 \\
\hline
\end{tabular}

Fuente: Elaboración propia, con información de la Comisión Nacional Bancaria y de Valores (CNBV).

\section{CONCLUSIONES}

Teorías recientes involucran el capital bancario como amortiguador del riesgo ya que algunos autores mencionan que al tener capital los bancos los hace más fuertes ante una probable quiebra por falta de liquidez. Pero eso no es del todo aceptado ya que, existen contrapuntos que el capital bancario alienta a los bancos a otorgar préstamos riesgosos (Coval y Thakor, 2005; Allen y Gale (2004), aunado a que el capital bancario impacta en la creación de liquidez en la economía. Las medidas de creación de liquidez propuestas por Deep y Schaefer (2004) y Berger y Bouwman (2009), se enfocan en representar el impulso que otorga el sector bancario a las economías, a través de la teoría de intermediación financiera, que como se mencionó, consta de captar depósitos líquidos para otorgar préstamos ilíquidos.

La aplicación de ambos modelos en la banca múltiple mexicana arrojó una creación de liquidez un tanto baja o bien una destrucción de la liquidez, como lo mencionaremos a continuación. Sin embargo, ambas medidas proporcionan un enfoque similar en la búsqueda de la medición de la creación de liquidez, al tomar la importancia del acaparamiento de los depósitos líquidos y encontrar su efecto en la colocación de los créditos que se otorgan en la economía.

A través de la medida brecha de liquidez o GAP LT de Deep y Schaefer (2004) la creación de liquidez de la banca múltiple mexicana mostro muy baja creación, con un promedio de 0.11 en cuanto el total de los activos del sector bancario, recordemos brecha se encuentra de -1 a 1 , en donde la máxima liquidez es 1. Esto quiere decir que la mayor parte de los préstamos otorgados o pasivos líquidos se encuentran financiados principalmente por capital o pasivos ilíquidos. En cuando a la medida de creación de liquidez "Cat Fat" propuesta por Berger y Bouwman (2009), el gremio bancario mexicano muestra una reducción o destrucción de la liquidez por $-843,803$ en promedio, teniendo en conclusión que la mayoría del financiamiento se encuentra probablemente a través de capital o pasivos ilíquidos, principalmente.

Con los resultados obtenidos, la hipótesis planteada en el presente artículo debiera alentar a la creación de liquidez por parte de la banca múltiple en México, como medida de evaluar la producción del sector, entendiéndose como qué tan eficaz es el sistema bancario, con el fin de dar un mayor empuje al crecimiento económico.

Sin embargo, existe la posibilidad que la creación de liquidez se vea afectada por el capital regulatorio. Por citar un ejemplo, posterior a la crisis financiera de 2008 presentada en estados unidos, el comité de Basilea ha exigido aumentar el capital 
regulatorio requerido de un $4 \% \quad 6 \%$ sobre los activos en riesgo y un $2.5 \%$ de capital fundamental dando un $8.5 \%$ (Basilea III, 2011). Aunado para México la CNBV, ha solicitado incrementar a $10.5 \%$ de capital regulatorio en base a los activos en riesgo, con el fin de proveer de un colchón para tiempos de crisis. Sin embargo, probablemente existe un impacto en la creación de liquidez observadas en el desarrollo del presente trabajo, proponiendo una línea de investigación futura de como el capital requerido afecta la creación de liquidez, esto sin afectar la estabilidad del sistema financiero. 


\section{REFERENCIAS}

Allen, F. Gale, D. (2004). Fianancial intermediaries and markets, Econometrica, 72(4), 1023-1061.

Allen, F., Carletti, E., \& Gale, D. (2009). Interbank market liquidity and central bank intervention. Journal of Monetary Economics, 56(5), 639-652.

Berger, A. N., Bouwman, C. H. (2009). Bank liquidity creation. The review of financial studies, 22(9), 3779-3837.

Berger, A. N., Bouwman, C. H. (2017). Bank liquidity creation, monetary policy, and financial crises. Journal of Financial Stability, 30, 139-155.

Berger, A. N., Boubakri, N., Guedhami, O., \& Li, X. (2019). Liquidity creation performance and financial stability consequences of Islamic banking: Evidence from a multinational study. Journal of Financial Stability, 44, 100692.

Bryant, John. (1980), A model of reserves, bank runs, and deposit insurance, Journal of Banking and Finance, 4, 335-344.

Coval, J. D., \& Thakor, A. V. (2005). Financial intermediation as a beliefs-bridge between optimists and pessimists. Journal of Financial Economics, 75(3), 535569.

Dang, V. D. (2021). How do bank characteristics affect the bank liquidity creation channel of monetary policy?. Finance Research Letters, 101984.

Deep, A., Schaefer, G. (2004). Are banks liquidity transformers? KSG Working Paper

No. RWP04-022, Available at SSRN: https://ssrn.com/abstract=556289 or http://dx.doi.org/10.2139/ssrn.556289

Detriagache, E. Rajan, R. (2008), The real effects of banking crises, Journal of Financial Intermediation, 17, 89-112.

Diamond, W. Dybvig, P. (1983), Bank runs, deposit insurance, and liquidity, Journal of Political Economy 91: 401-419.

Diamond, W., 1984, Financial intermediation and delegated monitoring, Review of Economic Studies, 51, 393-414.

Diamond, D. W., \& Rajan, R. G. (2000). A theory of bank capital. the Journal of Finance, 55(6), 2431-2465.

Kelly, B., Pástor, L., \& Veronesi, P. (2016). The price of political uncertainty: Theory and evidence from the option market. The Journal of Finance, 71(5), 2417-2480.

Le, T. (2019). The interrelationship between liquidity creation and bank capital in Vietnamese banking. Managerial Finance. Vol. 45 No. 2, pp. 331-347. https://doi.org/10.1108/MF-09-2017-0337

Le, T. D., Pham, X. T. (2021). The inter-relationships among liquidity creation, bank capital and credit risk: evidence from emerging Asia-Pacific economies. Managerial Finance. Vol. ahead-of-print No. ahead-of-print. https://doi.org/10.1108/MF-04-2020-0189

Pana, E., Park, J., \& Query, T. (2010). The impact of bank mergers on liquidity creation. Journal of Risk Management in Financial Institutions, 4(1), 74-96.

Pastor, L, Pietro V, (2013), Political Uncertainty and Risk Premia, Journal of Financial Economics 110, 520-545. 